\title{
COMMON PRINCIPAL COMPONENTS MODEL FOR SYMBOLIC DATA
}

\author{
Kotoe Katayama, Hiroyuki Minami ${ }^{\dagger}$ and Masahiro Mizuta ${ }^{\dagger}$
}

\begin{abstract}
This paper proposes a dimension reduction technique in the framework of symbolic data analysis (SDA). Recent advances in technology have increased the complexity of datasets, and today, their size is much larger than it was in the past decade. Most statistical methods do not have sufficient power to analyze these datasets. SDA was proposed by Diday at the end of the 1980s and is a new approach for analyzing huge and complex data.

SDA examines "symbolic data", which consist of concepts. A concept consists of not only values but also "higher-level units" such as an interval and a distribution. Their combination can also be represented as a kind of a concept. This implies that complex data can be formally handled in the framework of SDA. However, there are very few studies based on this simple idea. Therefore, practical methods should be developed to apply this idea to solve problems in the real world. In this study, we focus on the case in which a concept contains some subsets (the concept acts as a typical complex dataset) and develop a new method to analyze this dataset directly using SDA.

In this paper, we propose a dimension reduction technique in the framework of SDA, especially for a group structure, and introduce a numerical example.
\end{abstract}

\section{Introduction}

Conventional data analysis usually can handle scalars, vectors and matrices. However, lately, some datasets have grown beyond the framework of conventional data analysis. In this study, we attempted to extract useful information from such datasets.

Symbolic data analysis (SDA) proposed by Diday (1988) is an approach for analyzing new types of datasets. "Symbolic data" consist of a concept that is described by intervals, distributions, etc. as well as by numerical values. The use of SDA enriches data description, and it can handle highly complex datasets.

One of the complexities in current datasets is a nested structure. For example, consider a dataset that contains some subsets. Here, if we assume the subsets in advance, we say that the dataset has a group structure. If we analyze such a dataset using a conventional technique, we might lose some information because the analysis might reduce the dimension of the dataset without maintaining its original structure. Therefore, a new idea to analyze a group structure without the loss of information is required.

Many researchers have proposed extensions of conventional data analysis techniques to the framework of SDA. Dimension reduction is an important technique, and principal

\footnotetext{
*Human Genome Center, Institute of Medical Science, University of Tokyo, 4-6-1 Shirokanedai, Minato-ku, Tokyo 108-8639, Japan E-mail: k-kata@ims.u-tokyo.ac.jp

${ }^{\dagger}$ Information Initiative Center, Hokkaido University, N 11 W 5, Kita-ku, Sapporo 060-0811, Japan

Key words: Dimension reduction; Complex data; Group structure
} 
components analysis (PCA) is one of the typical methods that uses this technique. PCA for symbolic data has been studied previously, but most of these studies assume that a concept is an interval value and any set of concepts does not have group structures. Therefore, we focus on a concept that is described by distribution and contains a group structure. As a conventional data analysis technique, Flury (1984) proposed common principal components (CPC) model, which is an extension of PCA to the data that contain some group structures. We extend this idea to the framework of SDA.

In this paper, we propose a dimension reduction technique for group structures as a variation of the CPC model. We investigate the availability of our proposed technique through a numerical simulation.

\section{Common principal components model for symbolic data analysis}

\subsection{Common principal components model}

PCA is one of the well-known techniques in multivariate analysis. It is based on the reparameterization of a single covariance matrix $\Psi$, and the new parameters are the eigenvectors and the eigenvalues of $\Psi$.

The CPC model is an extension of PCA to the data that consist of several groups. The development of this model was motivated by biometrical applications, where patterns of principal components among different species were quite similar. It is assumed that $\mathbf{X}_{i}(i=1, \ldots, k)$ are $p$-variate random vectors and that $p \times p$ covariance matrices $\Psi_{1}, \ldots, \Psi_{k}$ of $k$ populations are positive definite. The model is defined as

$$
\Psi_{i}=\boldsymbol{\beta} \Lambda_{i} \boldsymbol{\beta}^{\prime}, \quad i=1, \ldots, k
$$

where $\Lambda_{i}=\operatorname{diag}\left(\lambda_{i 1}, \ldots, \lambda_{i p}\right)$ and $\boldsymbol{\beta}$ consists of the coefficients of the common principal components and is represented by an orthogonal $p \times p$ matrix.

In the model, the condition on the covariance matrices is simple: all covariance matrices are diagonalized by the same orthogonal matrix.

\subsection{The model and its parameter estimation}

In this section, we propose a common principal components model for SDA (CPCSD model). The CPC model can handle data that consist of several groups, but all of the resulting groups have the same principal components. As a precondition to the CPC model, all groups need to have the same covariance matrices. The CPCSD model can handle data that consist of several groups, in addition, all the covariance matrices of the groups do not need to have similar covariance matrices.

We assume that each concept is independently $p$-dimensional normal distributed as $N_{p}\left(\boldsymbol{\mu}_{i}, \Psi_{i}\right)$, where $i$ is an index of the concept, $\boldsymbol{\mu}_{i} \in \Re^{p}$, and all $\Psi_{i}$ are positive definite, and that the concepts are classified into groups based on the eigenvectors.

Let $k$ be the number of concepts and $r$ be the number of groups. We assume that group $g(g=1, \ldots, r)$ consists of $q_{g}+1$ concepts.

The CPCSD model is defined as

$$
\Psi_{i}=\boldsymbol{\beta}_{i} \Lambda_{i} \boldsymbol{\beta}_{i}^{\prime} \text { and } \mathbf{R B}=\mathbf{0},
$$

where $\boldsymbol{\beta}_{i}=\left(\beta_{i 1}, \ldots, \beta_{i p}\right)$ is a $p \times p$ orthogonal matrix and consists of eigenvectors of $\Psi_{i}$. $\Lambda_{i}=\operatorname{diag}\left(\lambda_{i 1}, \ldots, \lambda_{i p}\right), \lambda_{i j}>0, j=1, \ldots, p . \mathbf{B}=\left[\boldsymbol{\beta}_{1}^{\prime}, \ldots, \boldsymbol{\beta}_{r}^{\prime}, \boldsymbol{\beta}_{r+1}^{\prime}, \ldots, \boldsymbol{\beta}_{r+q_{1}}^{\prime}, \boldsymbol{\beta}_{r+q_{1}+1}^{\prime} \ldots\right.$, $\left.\boldsymbol{\beta}_{r+q_{1}+q_{2}}^{\prime}, \ldots, \boldsymbol{\beta}_{r+q_{1}+\ldots+q_{r-1}+1}^{\prime}, \ldots, \boldsymbol{\beta}_{r+q_{1}+\ldots+q_{r}}^{\prime}\right]^{\prime}$. In this model, we assume that group $g$ consists of the concepts whose eigenvectors of covariance matrices are $\boldsymbol{\beta}_{g}^{\prime}$ and $\boldsymbol{\beta}_{r+q_{1}+\ldots+q_{g-1}+1}^{\prime}$, 
$\ldots, \boldsymbol{\beta}_{r+q_{1}+\ldots+q_{g}}^{\prime} . \mathbf{R}$ is a given $p\left(q_{1}+\cdots+q_{r}\right) \times p k$ matrix, where $q_{1}+\cdots+q_{r}=k-r$.

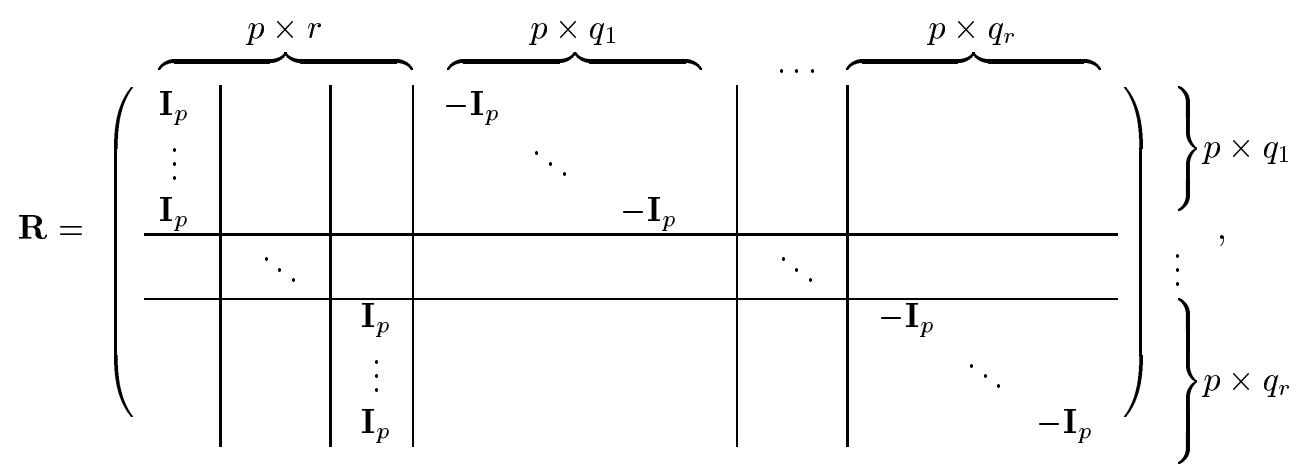

where $\mathbf{I}_{p}$ is a $p \times p$ identity matrix.

We use the maximum likelihood method to obtain the maximum likelihood estimations of $\boldsymbol{\beta}_{i}$ and $\Lambda_{i}$. We take $n_{i}$ samples from concept $i$. It is assumed that the sample covariance matrices are $\mathbf{S}_{i}(i=1, \ldots, k)$, which are independently Wishart distributed as $W_{p}\left(n_{i}-\right.$ $\left.1, \Psi_{i} /\left(n_{i}-1\right)\right)$. Because each concept is independently $p$-dimensional normal distributed as $N_{p}\left(\boldsymbol{\mu}_{i}, \Psi_{i}\right)$, if the sample size $n_{i}-1>p$, the distribution of $\mathbf{S}_{i}$ is Wishart distributed (Muirhead, 1982).

Given $\mathbf{S}_{1}, \ldots, \mathbf{S}_{k}$, the joint likelihood function of $\Psi_{1}, \ldots, \Psi_{k}$ is

$$
L\left(\Psi_{1}, \ldots, \Psi_{k}\right)=C \times \prod_{i=1}^{k}\left\{\exp \left[\operatorname{tr}\left(-\frac{n_{i}}{2} \Psi_{i}^{-1} \mathbf{S}_{i}\right)\right]\right\}\left(\operatorname{det} \Psi_{i}\right)^{-\frac{n_{i}}{2}},
$$

where $C$ does not depend on $\Psi_{i}$. Instead of maximizing the likelihood function, we minimize the function.

$$
g\left(\Psi_{1}, \ldots, \Psi_{k}\right)=\log C-\log L\left(\Psi_{1}, \ldots, \Psi_{k}\right) .
$$

The log-likelihood function is

$$
\log L\left(\Psi_{1}, \ldots, \Psi_{k}\right)=\log C-\frac{1}{2} \sum_{i=1}^{k} n_{i}\left[\operatorname{tr}\left(\Psi_{i}^{-1} \mathbf{S}_{i}\right)+\log \left(\operatorname{det} \Psi_{i}\right)\right]
$$

Then, we use

$$
\begin{aligned}
& \log L\left(\boldsymbol{\beta}_{1}, \ldots, \boldsymbol{\beta}_{k}, \Lambda_{1}, \ldots, \Lambda_{k}\right) \\
& =\log C-\frac{1}{2} \sum_{i=1}^{k} n_{i}\left\{\operatorname{tr}\left[\left(\boldsymbol{\beta}_{i} \Lambda_{i} \boldsymbol{\beta}_{i}^{\prime}\right)^{-1} \mathbf{S}_{i}\right]+\log \left(\operatorname{det} \boldsymbol{\beta}_{i} \Lambda_{i} \boldsymbol{\beta}_{i}^{\prime}\right)\right\} \\
& \quad=\log C-\frac{1}{2} \sum_{i=1}^{k} \sum_{j=1}^{p} n_{i}\left(\frac{\beta_{i j}^{\prime} \mathbf{S}_{i} \beta_{i j}}{\lambda_{i j}}+\log \lambda_{i j}\right)
\end{aligned}
$$


From the definition of the CPCSD model,

$$
\begin{gathered}
\log L\left(\boldsymbol{\beta}_{1}, \ldots, \boldsymbol{\beta}_{k}, \Lambda_{1}, \ldots, \Lambda_{k}\right) \\
=\log C-\frac{1}{2} \sum_{i=1}^{r} \sum_{j=1}^{p} n_{i}\left(\frac{\beta_{i j}^{\prime} \mathbf{S}_{i} \beta_{i j}}{\lambda_{i j}}+\log \lambda_{i j}\right)-\frac{1}{2} \sum_{i=r+1}^{k} \sum_{j=1}^{p} n_{i}\left(\frac{\beta_{i j}^{\prime} \mathbf{S}_{i} \beta_{i j}}{\lambda_{i j}}+\log \lambda_{i j}\right) \\
=\log C-\frac{1}{2} \sum_{i=1}^{r} \sum_{j=1}^{p}\left\{\left(n_{i} \log \lambda_{i j}+\sum_{l=m_{i}}^{h_{i}} n_{r+l} \log \lambda_{r+l \cdot j}\right)\right. \\
\left.\quad+\beta_{i j}^{\prime}\left(\frac{n_{i} \mathbf{S}_{i}}{\lambda_{i j}}+\sum_{l=m_{i}}^{h_{i}} \frac{n_{r+l} \mathbf{S}_{r+l}}{\lambda_{r+l \cdot j}}\right) \beta_{i j}\right\}
\end{gathered}
$$

where $q_{0}=0, m_{i}=1+\sum_{u=1}^{i} q_{u-1}$, and $h_{i}=\sum_{u=1}^{i} q_{u}$. Then,

$$
\begin{aligned}
g\left(\Psi_{1}, \ldots, \Psi_{k}\right) & =g\left(\boldsymbol{\beta}_{1}, \ldots, \boldsymbol{\beta}_{r}, \Lambda_{1}, \ldots, \Lambda_{k}\right) \\
=\frac{1}{2} \sum_{i=1}^{r} \sum_{j=1}^{p}\left\{\left(n_{i} \log \lambda_{i j}+\sum_{l=m_{i}}^{h_{i}} n_{r+l} \log \lambda_{r+l \cdot j}\right)\right. & \\
& \left.+\beta_{i j}^{\prime}\left(\frac{n_{i} \mathbf{S}_{i}}{\lambda_{i j}}+\sum_{l=m_{i}}^{h_{i}} \frac{n_{r+l} \mathbf{S}_{r+l}}{\lambda_{r+l \cdot j}}\right) \beta_{i j}\right\} .
\end{aligned}
$$

The function $g$ is to be minimized under the conditions

$$
\beta_{i d}^{\prime} \beta_{i j}=\delta_{d j}
$$

where $d=1, \ldots, p$ and $\delta_{d j}$ is the Kronecker delta function.

Thus, we attempt to minimize the Lagrange function

$$
\begin{gathered}
\operatorname{Lag}\left(\boldsymbol{\beta}_{1}, \ldots, \boldsymbol{\beta}_{r}, \Lambda_{1}, \ldots, \Lambda_{k}, \zeta_{11}, \ldots, \zeta_{r p}, \zeta_{121}, \ldots, \zeta_{r p(p-1)}\right) \\
=\frac{1}{2} \sum_{i=1}^{r} \sum_{j=1}^{p}\left\{\left(n_{i} \log \lambda_{i j}+\sum_{l=m_{i}}^{h_{i}} n_{r+l} \log \lambda_{l j}\right)\right. \\
\left.+\beta_{i j}^{\prime}\left(\frac{n_{i} \mathbf{S}_{i}}{\lambda_{i j}}+\sum_{l=m_{i}}^{h_{i}} \frac{n_{r+l} \mathbf{S}_{l}}{\lambda_{l j}}\right) \beta_{i j}\right\} \\
-\frac{1}{2} \sum_{i=1}^{r} \sum_{j=1}^{p} \zeta_{i j}\left(\beta_{i j}^{\prime} \beta_{i j}-1\right)-\sum_{i=1}^{r} \sum_{j=2}^{p} \sum_{d=1}^{j-1} \zeta_{i j d}\left(\beta_{i d}^{\prime} \beta_{i j}\right) .
\end{gathered}
$$

Taking partial derivatives with respect to $\lambda_{i a}$, we get

$$
\frac{\partial L a g}{\partial \lambda_{i a}}=\frac{1}{2}\left(\frac{n_{i}}{\lambda_{i a}}-\frac{n_{i} \beta_{i a}^{\prime} \mathbf{S}_{i} \beta_{i a}}{\lambda_{i a}^{2}}\right),
$$

and setting these derivatives to 0 yields

$$
\lambda_{i a}=\beta_{i a}^{\prime} \mathbf{S}_{i} \beta_{i a}, \quad i=1, \ldots, r, \quad a=1, \ldots, p .
$$


In addition, taking partial derivatives with respect to $\lambda_{r+l \cdot a}$, we get

$$
\frac{\partial L a g}{\partial \lambda_{r+l \cdot a}}=\frac{1}{2}\left(\frac{n_{r+l}}{\lambda_{r+l \cdot a}}-\frac{n_{r+l} \beta_{i a}^{\prime} \mathbf{S}_{r+l} \beta_{i a}}{\lambda_{r+l \cdot a}^{2}}\right),
$$

and setting these derivatives to 0 yields

$$
\lambda_{r+l \cdot a}=\beta_{i a}^{\prime} \mathbf{S}_{r+l} \beta_{i a} .
$$

To achieve the minimization of (1), we take partial derivatives with respect to $\beta_{i a}$ as follows:

$$
\begin{aligned}
\frac{\partial L a g}{\partial \beta_{i a}} & =\frac{1}{2}\left[2 \frac{n_{i} \mathbf{S}_{i}}{\lambda_{i a}} \beta_{i a}+2 \sum_{l=m_{i}}^{h_{i}} \frac{n_{r+l} \mathbf{S}_{r+l}}{\lambda_{r+l \cdot a}} \beta_{i a}\right]-\frac{1}{2} \cdot 2 \zeta_{i a} \beta_{i a}-\sum_{d=1}^{a} \zeta_{i a d} \beta_{i d}-\sum_{j=a+1}^{p} \zeta_{i j a} \beta_{i j} \\
& =\left(\frac{n_{i} \mathbf{S}_{i}}{\lambda_{i a}}+\sum_{l=m_{i}}^{h_{i}} \frac{n_{r+l} \mathbf{S}_{r+l}}{\lambda_{r+l \cdot a}}\right) \beta_{i a}-\zeta_{i a} \beta_{i a}-\sum_{d=1}^{a} \zeta_{i a d} \beta_{i d}-\sum_{j=a+1}^{p} \zeta_{i j a} \beta_{i j} .
\end{aligned}
$$

We set $(2)$ to 0 and multiply it by $\beta_{i j}^{\prime}(j>a)$ from the left, and thus, we get

$$
\beta_{i j}^{\prime}\left(\frac{n_{i} \mathbf{S}_{i}}{\lambda_{i a}}+\sum_{l=m_{i}}^{h_{i}} \frac{n_{r+l} \mathbf{S}_{r+l}}{\lambda_{r+l \cdot a}}\right) \beta_{i a}=\zeta_{i j a} .
$$

Similarly, we also multiply it by $\beta_{i j}^{\prime}(a>j)$ from the right and swap $j$ and $a$; thus, we get

$$
\beta_{i a}^{\prime}\left(\frac{n_{i} \mathbf{S}_{i}}{\lambda_{i j}}+\sum_{l=m_{i}}^{h_{i}} \frac{n_{r+l} \mathbf{S}_{r+l}}{\lambda_{r+l \cdot j}}\right) \beta_{i j}=\zeta_{i a j} .
$$

From (3) and (4), we get

$$
\beta_{i j}^{\prime}\left[\frac{\lambda_{i j}-\lambda_{i a}}{\lambda_{i j} \lambda_{i a}} n_{i} \mathbf{S}_{i}+\sum_{l=m_{i}}^{h_{i}} \frac{\lambda_{r+l \cdot j}-\lambda_{r+l \cdot a}}{\lambda_{r+l \cdot j} \lambda_{r+l \cdot a}} n_{r+l} \mathbf{S}_{r+l}\right] \beta_{i a}=0 .
$$

By using the maximum likelihood method, we obtain the following likelihood equation system:

$$
\left\{\begin{array}{l}
\beta_{i j}^{\prime} \beta_{i j}=1 \\
\beta_{i b}^{\prime} \beta_{i j}=0,(b \neq j) \\
\lambda_{i a}=\beta_{i a}^{\prime} \mathbf{S}_{i} \beta_{i a}, \\
\lambda_{r+l \cdot a}=\beta_{i a}^{\prime} \mathbf{S}_{r+l} \beta_{i a}, \\
\beta_{i j}^{\prime}\left[d \frac{\lambda_{i j}-\lambda_{i a}}{\lambda_{i j} \lambda_{i a}} n_{i} \mathbf{S}_{i}+\sum_{l=m_{i}}^{h_{i}} \frac{\lambda_{r+l \cdot j}-\lambda_{r+l \cdot a}}{\lambda_{r+l \cdot j} \lambda_{r+l \cdot a}} n_{r+l} \mathbf{S}_{r+l}\right] \beta_{i a}=0 .
\end{array}\right.
$$

We solve the equation system and get the maximum likelihood of $\beta_{i j}, \lambda_{i a}$, and $\lambda_{r+l \cdot a}$. The numerical computation for this equation system was developed by Flury and Neuenschwander (1995). 


\section{Visualization of similarities between groups}

In this section, we define similarities between groups using principal components and visualize the structure among all groups using multidimensional scaling (MDS).

Suppose that two groups $m, n$ have the following principal components : $\left\{\beta_{1}^{(m)}, \ldots, \beta_{h}^{(m)}\right\}$, $\left\{\beta_{1}^{(n)}, \ldots, \beta_{h}^{(n)}\right\}$, where $h<p, \beta_{i}^{(m)} \in \Re^{p}, \beta_{j}^{(n)} \in \Re^{p}, \beta_{i}^{(m)^{\prime}} \beta_{j}^{(m)}=\delta_{i j}^{(m)}$, and $\beta_{i}^{(n)^{\prime}} \beta_{j}^{(n)}=$ $\delta_{i j}^{(n)}$.

Their linear combinations are

$$
\begin{aligned}
& \mathbf{x}=a_{1} \beta_{1}^{(m)}+\cdots+a_{h} \beta_{h}^{(m)}=\sum_{i=1}^{h} a_{i} \beta_{i}^{(m)} \\
& \mathbf{y}=b_{1} \beta_{1}^{(n)}+\cdots+b_{h} \beta_{h}^{(n)}=\sum_{j=1}^{h} b_{j} \beta_{j}^{(n)}
\end{aligned}
$$

where $a_{1}, \ldots, a_{h}$ and $b_{1}, \ldots, b_{h}$ are scalars, $\sum_{i=1}^{h} a_{i}^{2}=1$, and $\sum_{j=1}^{h} b_{j}^{2}=1$. We assume that $\theta_{\mathbf{x y}}$ is the angle between $\mathbf{x}$ and $\mathbf{y}$. Then,

$$
\begin{aligned}
\cos \theta_{\mathbf{x y}} & =\frac{\mathbf{x}^{\prime} \mathbf{y}}{|\mathbf{x}||\mathbf{y}|}=\mathbf{x}^{\prime} \mathbf{y}=\sum_{i=1}^{h} a_{i} \beta_{i}^{(m)^{\prime}} \sum_{j=1}^{h} b_{j} \beta_{j}^{(n)}=\sum_{i=1}^{h} \sum_{j=1}^{h} a_{i} b_{j} \beta_{i}^{(m)^{\prime}} \beta_{j}^{(n)} \\
& =\left(a_{1}, \ldots, a_{h}\right)\left(\begin{array}{ccc}
\beta_{1}^{(m)^{\prime}} \beta_{1}^{(n)} & \cdots & \beta_{1}^{(m)^{\prime}} \beta_{h}^{(n)} \\
\vdots & & \vdots \\
\beta_{h}^{(m)^{\prime}} \beta_{1}^{(n)} & \cdots & \beta_{h}^{(m)^{\prime}} \beta_{h}^{n}
\end{array}\right)\left(\begin{array}{c}
b_{1} \\
\vdots \\
b_{h}
\end{array}\right) \\
& =\mathbf{a}^{\prime} \mathbf{F} \mathbf{b}
\end{aligned}
$$

where $\mathbf{a}^{\prime}=\left(a_{1}, \ldots, a_{h}\right), \mathbf{b}^{\prime}=\left(b_{1}, \ldots, b_{h}\right)$, and $\mathbf{F}=\left(\beta_{i}^{(m)^{\prime}} \beta_{j}^{(n)}\right)$. We define the maximum value of $\cos \theta_{\mathbf{x y}}$ as the similarity between the groups $m, n$ and denote it as $s_{m n}$.

Next, we calculate $s_{m n} a$. We apply singular value decomposition to $\mathbf{F}$ and get

$$
\mathbf{F}=\mathbf{U} \Delta \mathbf{V}^{\prime},
$$

where $\mathbf{U}^{\prime} \mathbf{U}=\mathbf{U U}^{\prime}=\mathbf{I}_{h}, \mathbf{V}^{\prime} \mathbf{V}=\mathbf{V V}^{\prime}=\mathbf{I}_{h}$,

$$
\Delta=\left(\begin{array}{cc}
\Delta_{r} & 0 \\
0 & 0
\end{array}\right), \quad \Delta_{r}=\left(\begin{array}{ccc}
\lambda_{1} & & 0 \\
& \ddots & \\
0 & & \lambda_{r}
\end{array}\right) \text {, and } \lambda_{1} \geq \lambda_{2} \geq \cdots \geq \lambda_{r} \geq 0 .
$$

$\lambda_{1}, \ldots, \lambda_{r}$ are singular values of $\mathbf{F}$ and equal to square roots of non-zero eigenvalues of $\mathbf{F}^{\prime} \mathbf{F}$. Substituting (6) into (5), we get

$$
\cos \theta_{\mathbf{x y}}=\mathbf{x}^{\prime} \mathbf{y}=\mathbf{a}^{\prime} \mathbf{F} \mathbf{b}=\mathbf{a}^{\prime} \mathbf{U} \Delta \mathbf{V}^{\prime} \mathbf{b}=\mathbf{W}^{\prime} \Delta \mathbf{Z},
$$

where

$$
\begin{aligned}
& \mathbf{W}^{\prime}=\mathbf{a}^{\prime} \mathbf{U}, \mathbf{Z}=\mathbf{V}^{\prime} \mathbf{b}, \\
& \left\|\mathbf{W}^{\prime}\right\|^{2}=\left\|\mathbf{a}^{\prime} \mathbf{U}\right\|^{2}=\mathbf{a}^{\prime} \mathbf{U} \mathbf{U}^{\prime} \mathbf{a}=\mathbf{a}^{\prime} \mathbf{I}_{h} \mathbf{a}=\mathbf{a}^{\prime} \mathbf{a}=1, \text { and } \\
& \left\|\mathbf{Z}^{\prime}\right\|^{2}=\left\|\mathbf{b}^{\prime} \mathbf{V}\right\|^{2}=\mathbf{b}^{\prime} \mathbf{V} \mathbf{V}^{\prime} \mathbf{b}=\mathbf{b}^{\prime} \mathbf{I}_{h} \mathbf{b}=\mathbf{b}^{\prime} \mathbf{b}=1 .
\end{aligned}
$$


Therefore, we get $\sum_{i=1}^{r} W_{i}^{2}=1, \sum_{i=1}^{r} V_{i}^{2}=1$. Thus, (7) becomes

$$
\cos \theta_{\mathbf{x y}}=\mathbf{x}^{\prime} \mathbf{y}=\lambda_{1} W_{1} Z_{1}+\cdots+\lambda_{r} W_{r} Z_{r}=\sum_{i=1}^{r} \lambda_{i} W_{i} Z_{i} .
$$

The maximum value of (8) is $s_{m n}$. We set a similarity matrix $S$, where $s_{m n}=s_{n m}(m, n=$ $1, \ldots, r)$, and if $m=n, s_{m n}=1$.

To place the similarity matrix $S$ in a plot, we use Torgerson's metric MDS. By using Torgerson's metric MDS, we can project concepts into a two-dimensional plot and also visualize the structure of concepts within a group.

\section{Numerical simulation}

As a typical example for the implementation of the CPCSD model, consider a dataset that is divided into some groups including concepts. The covariance matrices in a group are similar but not identical.

Now, we have 15 concepts, represented by four-dimensional normal distributions. We can classify them into 5 groups: A, B, C, D, and E. To verify the effectiveness of the visualization of a relationship between groups, we intentionally regard the eigenvectors of $\mathrm{A}$ and $\mathrm{B}$ to be dissimilar and those of C, D, and E to be similar. Each group consists of 3 concepts; for example, Group A has the concepts A1, A2, and A3. The example dataset is as follows.

$$
\begin{aligned}
& A 1 \sim N_{4}\left(\left(\begin{array}{l}
3.013 \\
2.964 \\
1.982 \\
1.991
\end{array}\right),\left(\begin{array}{rrrr}
3.985 & -0.097 & 0.044 & -0.061 \\
-0.097 & 2.981 & -0.093 & 0.022 \\
0.044 & -0.093 & 2.034 & 0.079 \\
-0.062 & 0.022 & 0.079 & 1.026
\end{array}\right)\right) \\
& A 2 \sim N_{4}\left(\left(\begin{array}{l}
4.035 \\
4.127 \\
3.998 \\
3.979
\end{array}\right),\left(\begin{array}{rrrr}
4.097 & 0.063 & 0.005 & 0.053 \\
0.063 & 2.956 & -0.025 & 0.045 \\
0.005 & -0.025 & 2.127 & 0.029 \\
0.053 & 0.045 & 0.029 & 0.967
\end{array}\right)\right) \\
& A 3 \sim N_{4}\left(\left(\begin{array}{l}
5.006 \\
2.032 \\
1.997 \\
5.012
\end{array}\right),\left(\begin{array}{rrrr}
3.860 & 0.094 & -0.050 & 0.057 \\
0.094 & 3.138 & 0.065 & -0.074 \\
-0.050 & 0.065 & 1.947 & 0.059 \\
0.057 & -0.074 & 0.059 & 0.990
\end{array}\right)\right) \\
& B 1 \sim N_{4}\left(\left(\begin{array}{l}
3.043 \\
2.982 \\
2.004 \\
2.029
\end{array}\right),\left(\begin{array}{rrrr}
0.935 & -0.014 & -0.074 & -0.030 \\
-0.014 & 2.272 & -0.567 & -0.088 \\
-0.074 & -0.567 & 3.450 & 0.220 \\
-0.030 & -0.088 & 0.220 & 2.963
\end{array}\right)\right) \\
& B 2 \sim N_{4}\left(\left(\begin{array}{l}
4.003 \\
3.941 \\
3.992 \\
4.004
\end{array}\right),\left(\begin{array}{rrrr}
0.947 & 0.066 & -0.040 & -0.028 \\
0.066 & 2.425 & -0.869 & -0.002 \\
-0.040 & -0.869 & 3.489 & 0.107 \\
-0.028 & -0.002 & 0.107 & 2.974
\end{array}\right)\right) \\
& B 3 \sim N_{4}\left(\left(\begin{array}{l}
4.975 \\
1.984 \\
1.986 \\
4.998
\end{array}\right),\left(\begin{array}{rrrr}
0.984 & -0.042 & 0.040 & -0.014 \\
-0.042 & 2.188 & -0.689 & -0.063 \\
0.040 & -0.689 & 3.754 & 0.019 \\
-0.014 & -0.063 & 0.019 & 2.940
\end{array}\right)\right) \\
& C 1 \sim N_{4}\left(\left(\begin{array}{l}
2.941 \\
2.965 \\
2.011 \\
2.068
\end{array}\right),\left(\begin{array}{rrrr}
2.346 & -0.071 & 0.473 & 0.664 \\
-0.071 & 4.144 & -0.055 & -0.091 \\
0.473 & -0.055 & 2.292 & 0.244 \\
0.664 & -0.090 & 0.244 & 1.233
\end{array}\right)\right)
\end{aligned}
$$


KATAYAMA, MINAMI and MIZUTA

$$
\begin{aligned}
& C 2 \sim N_{4}\left(\left(\begin{array}{l}
3.993 \\
4.012 \\
3.964 \\
3.992
\end{array}\right),\left(\begin{array}{rrrr}
2.353 & -0.018 & 0.541 & 0.558 \\
-0.018 & 3.856 & 0.152 & -0.133 \\
0.540 & 0.152 & 2.267 & 0.215 \\
0.558 & -0.133 & 0.215 & 1.195
\end{array}\right)\right) \\
& C 3 \sim N_{4}\left(\left(\begin{array}{l}
4.993 \\
2.012 \\
1.964 \\
4.993
\end{array}\right),\left(\begin{array}{rrrr}
2.252 & -0.091 & 0.407 & 0.714 \\
-0.091 & 4.245 & -0.018 & -0.052 \\
0.407 & -0.018 & 2.354 & 0.173 \\
0.714 & -0.052 & 0.173 & 1.215
\end{array}\right)\right) \\
& D 1 \sim N_{4}\left(\left(\begin{array}{l}
2.963 \\
3.021 \\
1.949 \\
2.064
\end{array}\right),\left(\begin{array}{rrrr}
4.134 & 0.008 & -0.041 & -0.213 \\
0.008 & 2.195 & -0.315 & 0.777 \\
-0.041 & -0.315 & 1.603 & 0.319 \\
-0.213 & 0.777 & 0.319 & 2.443
\end{array}\right)\right) \\
& D 2 \sim N_{4}\left(\left(\begin{array}{l}
4.063 \\
3.995 \\
3.991 \\
3.969
\end{array}\right),\left(\begin{array}{rrrr}
3.964 & -0.115 & 0.043 & 0.133 \\
-0.115 & 2.342 & -0.315 & 0.830 \\
0.043 & -0.315 & 1.301 & 0.367 \\
0.133 & 0.829 & 0.367 & 2.441
\end{array}\right)\right) \\
& D 3 \sim N_{4}\left(\left(\begin{array}{l}
5.048 \\
1.993 \\
1.973 \\
4.953
\end{array}\right),\left(\begin{array}{rrrr}
3.934 & 0.073 & -0.062 & 0.158 \\
0.073 & 1.962 & -0.314 & 0.746 \\
-0.062 & -0.314 & 1.345 & 0.372 \\
0.158 & 0.746 & 0.372 & 2.549
\end{array}\right)\right) \\
& E 1 \sim N_{4}\left(\left(\begin{array}{l}
2.933 \\
3.087 \\
1.987 \\
2.102
\end{array}\right),\left(\begin{array}{rrrr}
4.137 & 0.458 & 0.620 & -0.254 \\
0.458 & 2.185 & -0.228 & 0.714 \\
0.620 & -0.228 & 2.267 & -0.167 \\
-0.254 & 0.714 & -0.167 & 1.536
\end{array}\right)\right) \\
& E 2 \sim N_{4}\left(\left(\begin{array}{l}
3.998 \\
3.895 \\
3.992 \\
4.001
\end{array}\right),\left(\begin{array}{rrrr}
3.871 & 0.497 & 0.647 & -0.204 \\
0.497 & 2.289 & -0.172 & 0.838 \\
0.647 & -0.172 & 2.321 & -0.399 \\
-0.204 & 0.838 & -0.399 & 1.662
\end{array}\right)\right) \\
& E 3 \sim N_{4}\left(\left(\begin{array}{l}
4.984 \\
2.030 \\
1.892 \\
5.080
\end{array}\right),\left(\begin{array}{rrrr}
3.936 & 0.471 & 0.426 & -0.253 \\
0.471 & 2.244 & -0.239 & 0.770 \\
0.426 & -0.239 & 2.346 & -0.180 \\
-0.253 & 0.770 & -0.180 & 1.568
\end{array}\right)\right)
\end{aligned}
$$

We apply the CPCSD model to the dataset and obtain the common principal components as follows.

\begin{tabular}{|l|l|l|}
\hline Group A & PC1 & $(0.997,-0.098,0.026,-0.021)$ \\
& PC2 & $(0.068,0.997,0.019,-0.312)$ \\
\hline Group B & PC1 & $(0.028,0.358,-0.888,-0.289)$ \\
& PC2 & $(0.036,-0.927,-0.373,-0.957)$ \\
\hline Group C & PC1 & $(-0.072,0.994,-0.054,-0.051)$ \\
& PC2 & $(0.731,0.103,0.579,0.345)$ \\
\hline Group D & PC1 & $(-0.987,0.050,0.028,0.150)$ \\
& PC2 & $(-0.147,-0.648,-0.019,-0.747)$ \\
\hline Group E & PC1 & $(0.950,0.149,0.265,-0.063)$ \\
& PC2 & $(-0.036,-0.758,0.440,-0.481)$ \\
\hline
\end{tabular}


Common Principal Components Model for Symbolic Data

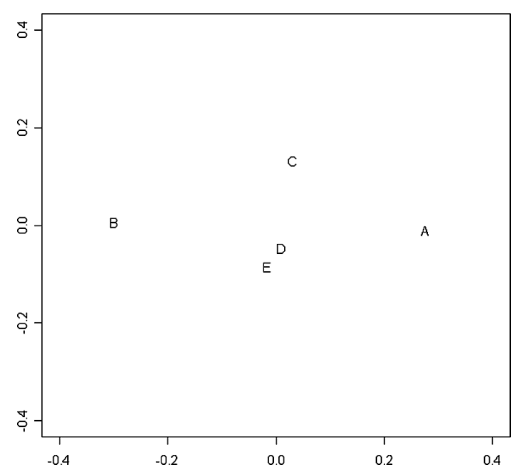

Fig. 1: MDS plot of 5 groups

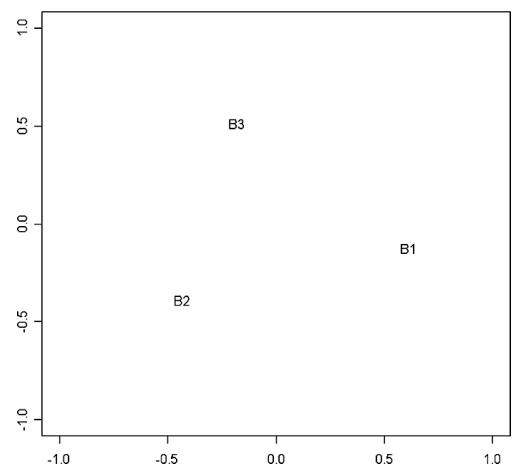

Fig. 3: MDS plot of group B

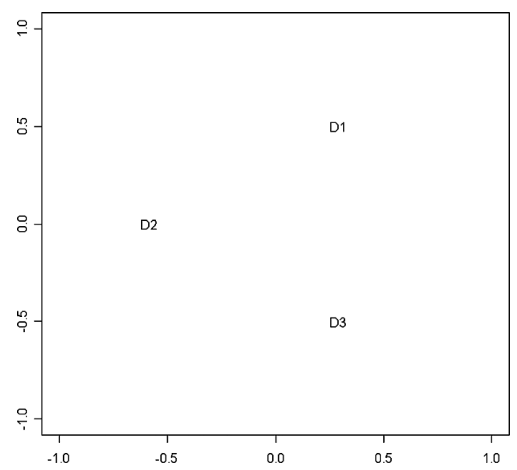

Fig. 5: MDS plot of group D

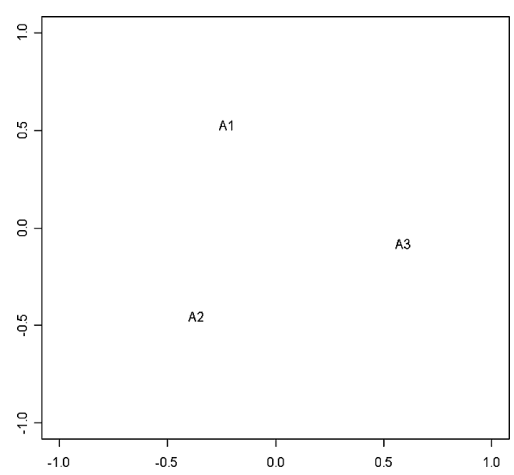

Fig. 2: MDS plot of group A

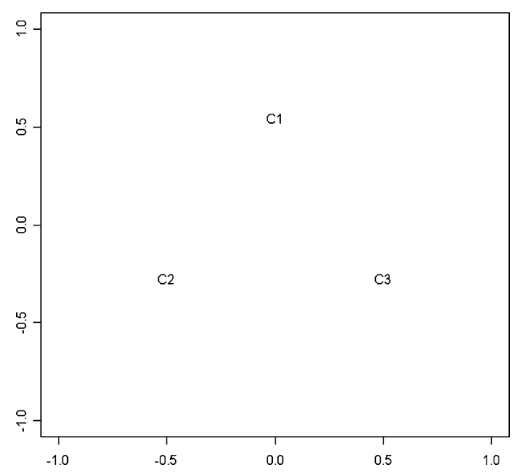

Fig. 4: MDS plot of group C

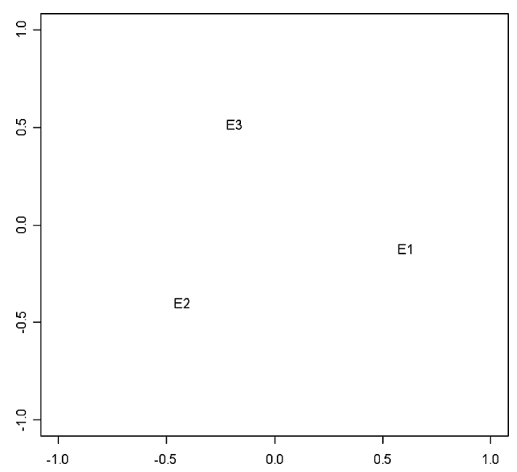

Fig. 6: MDS plot of group E 
KATAYAMA, MINAMI and MIZUTA

Now, we calculate the similarity matrix from these components.

\begin{tabular}{l|ccccc} 
& \multicolumn{6}{|c}{ GroupA } & GroupB & GroupC & GroupD & GroupE \\
\hline GroupA & 1.000 & 0.423 & 0.938 & 0.927 & 0.994 \\
GroupB & 0.423 & 1.000 & 0.770 & 0.813 & 0.910 \\
GroupC & 0.938 & 0.770 & 1.000 & 0.817 & 0.777 \\
GroupD & 0.927 & 0.813 & 0.817 & 1.000 & 0.936 \\
GroupE & 0.994 & 0.910 & 0.777 & 0.936 & 1.000
\end{tabular}

We apply MDS to this matrix and obtain the plot shown in Figure 1. In this simulation, we can maintain groups $\mathrm{C}, \mathrm{D}$, and $\mathrm{E}$ to be similar and the difference between groups $\mathrm{A}$ and $\mathrm{B}$ to be large. The difference between groups $\mathrm{A}$ and $\mathrm{C}$ is nearly equal to that between groups $\mathrm{A}$ and D and also between groups $\mathrm{A}$ and $\mathrm{E}$. The same result is true for groups B, C, D, and E. Among all the groups, groups D and E are very similar to each other. This result reflects the initial condition; we intentionally regarded the eigenvectors of $\mathrm{A}$ and $\mathrm{B}$ to be dissimilar and those of $\mathrm{C}, \mathrm{D}$, and $\mathrm{E}$ to be similar.

We also visualize the structure of the concepts within a group, as shown in Figure 2 to Figure 6. It is confirmed that the structure of the concepts is reproduced by the proposed method. The plots are almost the same as those of the sample datasets extracted from the corresponding concepts.

\section{Conclusion}

In this paper, we proposed a dimension reduction technique for the case in which the concept was described by distribution and contained group structures. We proposed the CPCSD model and obtained the likelihood equation system. We also defined similarities between groups using principal components and visualized the structure among groups. Through the simulation, we validated the effectiveness of our model.

In the future, we intend to apply this model to practical data and solve the associated problems. We also intend to generalize the CPCSD model to the case in which a concept is described by a distribution that belongs to the exponential family.

\section{REFERENCES}

Diday, E. (1988). The symbolic approach in clustering and related methods of data analysis. In Classification and Related Methods of Data Analysis, H.Bock (ed.), 673-684, Amsterdam: North-Holland.

Flury, B.D. (1984). Common principal components in K groups. Journal of the American Statistical Association 79, 892-898.

Flury, B.D. and Neuenschwander, B.E. (1995). Simultaneous diagonalization algorithm with applications in multivariate statistics. In Approximation and Computation, R.V.M. Zahar (ed.), 179-208, Basel: Brikhäuser.

Muirhead, R.J. (1982). Aspects of Multivariate Statistical Theory. New York: Wiley.

(Received: September 24, 2009, Accepted: October 20, 2010) 